\title{
Localized-active-space pair-density functional theory
}

Riddhish Pandharkar ${ }^{\mathrm{a}}$, Matthew R. Hermes ${ }^{\mathrm{a}}$, Christopher J. Cramer ${ }^{\mathrm{b}}$, Donald G. Truhlar ${ }^{\mathrm{b} *}$, and Laura Gagliardi ${ }^{\mathrm{a} *}$

a Department of Chemistry, Pritzker School of Molecular Engineering, James Franck Institute, Chicago Center for Theoretical Chemistry, The University of Chicago, 5735 S Ellis Ave., Chicago, IL 60637, United States

${ }^{\mathrm{b}}$ Department of Chemistry, Chemical Theory Center, and Minnesota Supercomputing Institute, University of Minnesota, Minneapolis, MN 55455-0431, United States

Corresponding authors: truhlar@umn.edu, 1gagliardi@uchicago.edu

\begin{abstract}
Accurate quantum chemical methods for the prediction of spin-state energy gaps for strongly correlated systems are computationally expensive and scale poorly with the size of the system. This makes calculations for many experimentally interesting molecules impractical even with abundant computational resources. In previous work, we have shown that the localized active space (LAS) self-consistent field (SCF) method is an efficient way to obtain multi-configuration SCF wave functions of comparable quality to the corresponding complete active space (CAS) ones. To obtain quantitative results, a post-SCF method is needed to estimate the complete correlation energy. One such method is multiconfiguration pair-density functional theory (PDFT), which calculates the energy based on the density and on-top pair density obtained from a multiconfiguration wave function. In this work we introduce localized-active-space pair-density functional theory, which uses a LAS wave function for subsequent PDFT calculations. The method is tested for computing spin-state energy gaps in conjugated organic molecules and bimetallic compounds and is shown to give results within $0.05 \mathrm{eV}$ of the corresponding CAS-PDFT results at a significantly lower cost.
\end{abstract}

\section{Introduction}


Quantum mechanical electronic structure methods for making quantitative predictions of the properties of molecules and materials often present a tradeoff between accuracy and cost in computing electron correlation. Many approaches have been developed to account for static and dynamic correlation at a reasonable computational cost. ${ }^{1-5}$ While there is not a unique way to decompose the electron correlation energy into these two contributions, ${ }^{6}$ the distinction remains useful for understanding the performance of various methods and improving them in systematic ways. One helpful key to distinguish these alternative correlation effects is provided by the concept of entanglement. ${ }^{7}$

Multiconfiguration self-consistent field (MCSCF) methods are often used as a first step when dealing with systems that have significant static correlation. The complete active space SCF (CASSCF) method expresses the wave function as a linear combination of all electronic configuration state functions that can be generated by allowing a set of active electrons to occupy a set of active orbitals in all possible ways that are consistent with selected space and spin symmetry choices. ${ }^{8}$ With practically affordable active space sizes, CASSCF recovers only a relatively small portion of the dynamic correlation energy, and post-SCF methods are needed to make quantitative predictions. CASPT2 is a popular post-CASSCF method that uses second-order perturbation theory to add dynamic correlation energy to the CASSCF energy, ${ }^{9}$ but accurate CASPT2 calculations are prohibitively expensive except for small molecules. ${ }^{10,11}$ One post-SCF treatment that is more affordable than CASPT2 and that has proven to be useful is multiconfiguration pair-density functional theory (MC-PDFT). ${ }^{12}$ Instead of computing perturbation-theory corrections to the wave function, the MC-PDFT method involves an expression for the total energy as a functional of the kinetic energy, electron density, and on-top pair density of an unmodified mutlticonfiguration wave function. ${ }^{13,14} \mathrm{MC}$ PDFT has been shown to give CASPT2-quality results for many quantities like spin-state energy gaps, excitation energies, and binding energies for a variety of systems. ${ }^{12,13,15,16}$

Although CAS-PDFT allows us to tackle larger systems than are affordable with CASPT2, obtaining the reference CASSCF wave function remains computationally challenging for large systems. MC-PDFT has also been successfully employed with other reference wave functions, such as RASSCF ${ }^{17}$ GASSCF, ${ }^{18-20}$ 
DMRG-SCF, ${ }^{21,22}$ and RASCI ${ }^{23}$ (where RAS, CAS, and DMRG denote respectively restricted active space, generalized active space, and density matrix renormalization group, and RASCI denotes restricted active space configuration interaction).

Although approximating the wave function using the above methods allows us to study increasingly large molecules and active spaces, they still suffer from the computational effort scaling exponentially with system size, and this limits their application. We recently proposed a method to obtain a new kind of multiconfiguration wave function called LASSCF. ${ }^{24,25}$ To understand the motivation for this method, we may categorize the types of interaction between subsystems by using the concept of entanglement. In many cases, a system may be theoretically decomposed into fragments with little entanglement between the fragments, but high static correlation within one or more of the fragments. LASSSCF is designed for this kind of system, and with LASSCF, the entire active space is decomposed in multiple unentangled active subspaces that are localized on user- defined fragments. Since these subspaces are unentangled, the wave function for each of them can be well approximated by treating the interaction with other subspaces as a mean field. This means that the method scales exponentially only with the size of each individual active subspace, while it scales linearly with the number of subspaces. The LASSCF energy is a variational upper bound to the corresponding CASSCF energy. ${ }^{24}$

In this work, we introduce a new method called localized-active-space pair-density functional theory (LASPDFT), which uses LASSCF wave functions as reference wave functions for PDFT calculations. We investigate if the modest sensitivity of the MC-PDFT energies to the 'quality' of the reference MCSCF wave function applies also to fragmented, unentangled active spaces. The new LAS-PDFT method is tested for calculating the spin-state energetics of conjugated organic molecules and bimetallic complexes. We investigate the differences in LASSCF and LAS-PDFT energies as compared to the corresponding CASSCF and CAS-PDFT energies, respectively. We also explore the effects of varying the sizes of the active spaces and basis sets.

\section{Theory and Computational Methods}


In this section we briefly review LASSCF and MC-PDFT. More detailed treatments of both these methods are already in the literature. ${ }^{12,16} 24,25$

We consider the case of multiple active subspaces and one inactive subspace. The inactive subspace is described by a closed-shell-singlet determinant. The LASSCF wave function is formulated as an antisymmetrized product of wave functions, $\psi$, of the $k$ various active subspaces, $A_{k}$, and the singledeterminant wave function $\Phi_{U}$ of the inactive electrons (the inactive subspace) as

$$
|\mathrm{LAS}\rangle=\left[\wedge_{k}\left|\psi_{A_{k}}\right\rangle\right] \wedge\left|\Phi_{U}\right\rangle
$$

where $\wedge$ denotes the antisymmetrized tensor product given mathematically by the Grassman wedge product. ${ }^{26}$ The wave function is optimized variationally to minimize the energy given by:

$$
E_{\mathrm{LAS}}=\langle\mathrm{LAS}|\widehat{H}| \mathrm{LAS}\rangle
$$

In practice a LASSCF calculation is initialized by orthogonalizing the atomic orbital basis using the metaLöwdin method ${ }^{27,28}$ and selecting the orthogonalized AOs of various collections of atoms as "fragment orbitals" of the groups on which the user chooses to localize the active subspaces. Then, either an initial guess of the active orbitals (which typically are delocalized over the entire molecule) is projected on to these fragment orbitals, or the fragment orbitals are projected onto the initial guess. (The latter projection leads to faster SCF convergence if the initial guess is very near to the converged result; otherwise the former is preferred.) In subsequent iterations, the energy is minimized with respect to orbitals and configuration interaction vectors without any constraint or any further orbital projection. The locality of the converged active orbitals is therefore due entirely to the initial guess. While this means there is no absolute guarantee that a physically meaningful LAS wave function can be obtained for any arbitrary combination of subspaces, we have observed that the orbitals deviate minimally from the initial guess provided the initial assignment to the fragments has been done reasonably well. This might not be the case when the subspaces are unphysical or when modeling some high-energy LAS state (e.g. a charge transfer from one subspace to 
another). The choices made for each system studied in this work are further elaborated in the discussion. As discussed in our previous work, LASSCF also allows the user to assign explicit local spin quantum numbers to different active subspaces. Since the wave function for each active subspace is optimized separately in the field of other subspaces, the user can choose any possible spin for each active subspace. The total LAS wave function, however, is not a spin eigenstate when multiple subspaces have non-singlet spins that are not mutually parallel (i.e., not all $M_{S}=+S$ or all $M_{S}=-S$, where $S$ and $M_{S}$ refer here to the spin and spin component quantum numbers of a subspace). We utilize the variational version of LASSCF described in Ref. ${ }^{24}$ throughout this work.

MC-PDFT calculates the total energy as ${ }^{12}$

$E_{\mathrm{MC}-\mathrm{PDFT}}[\rho, \Pi]=V_{\mathrm{NN}}+\left\langle\Psi^{\mathrm{MC}}|\widehat{T}| \Psi^{\mathrm{MC}}\right\rangle+\int \rho(\mathbf{r}) v_{\mathrm{Ne}}(\mathbf{r}) d \mathbf{r}+\iint \frac{\rho\left(\mathbf{r}_{1}\right) \rho\left(\mathbf{r}_{2}\right)}{r_{12}} d \mathbf{r}_{1} d \mathbf{r}_{2}+E_{\mathrm{ot}}[\rho, \Pi]$

where $V_{\mathrm{NN}}$ is the nuclear repulsion energy, $\left\langle\Psi^{\mathrm{MC}}|\widehat{T}| \Psi^{\mathrm{MC}}\right\rangle$ is the kinetic energy using calculated from the multiconfigurational reference wave function $\Psi^{\mathrm{MC}}, \int \rho(\mathbf{r}) v_{\mathrm{Ne}}(\mathbf{r}) d \mathbf{r}$ and $\iint \frac{\rho\left(\mathbf{r}_{1}\right) \rho\left(\mathbf{r}_{2}\right)}{r_{12}} d \mathbf{r}_{1} d \mathbf{r}_{2}$ are nuclearelectron attraction and classical Coulomb repulsion calculated from the density $(\rho)$ of $\Psi^{\mathrm{MC}}$, and $E_{\mathrm{ot}}$ is the on-top energy, which is a functional of $\rho$ and the and on-top pair density ( $\Pi$ ) of $\Psi^{\mathrm{MC}}$. As in Kohn-Sham theory, we do not know an exact functional form for the density functional, but various approximate functionals are available; the translated PBE (tPBE) functional is used in the present study.

In this work, we use LASSCF wave functions for $\Psi^{\mathrm{MC}}$. For deeper understanding, we not only calculate the LAS-PDFT energy, but also we decompose it into its components and compare them to the corresponding CAS-PDFT energy components.

We also compare the spin density ${ }^{29}$ and unpaired density ${ }^{30-32}$ of the underlying LASSCF wave function to those of the CASSCF wave function to get insight into the electronic structure on individual subspaces. For 
each atom we report the Mulliken spin density, obtained from the difference between the $\alpha$ and $\beta$ densities localized on each atom. The unpaired density on atom $A$, is

$$
u_{A}=\sum_{\mu \in A} \sum_{\nu}\left(D_{\mu \nu}-\sum_{\kappa \lambda} D_{\mu \kappa} S_{\kappa \lambda} D_{\lambda \nu}\right)
$$

where $\mathbf{S}$ is the overlap matrix, $\mathbf{D}$ is the spin-summed one-body reduced density matrix, and $\mu, v, \kappa$, and $\lambda$ are indices of basis functions centered on atoms A. This quantity gives the number of unpaired electrons of either spin, which is different from the spin density and can be used to detect unpaired spins in hyper open shells, such as open-shell singlets, doublets with three unpaired electrons, triplets with four unpaired electrons, etc. Unlike the spin density, the unpaired density of the entire system does not integrate to any specific number, it is bounded from below by the net spin density and from above by the total number of electrons.

All calculations reported in this work have been done on a locally modified version of the $m r h$ code. The 6-31G basis set was used for systems $1-3$, and the cc-pVDZ basis set was used for system 4 . Some bigger basis sets have also been tested for system 2, details of which can be found in section S5 along with other computational details. A sample input file for the LASSCF calculation for system 2 is provided in the electronic SI. Details about the active space and their partitioning into subspaces for each system are reported in their respective section.

\section{Results and Discussion}

The performance of LAS-PDFT was tested by computing absolute energies and spin-state energy gaps for four molecules and comparing them to the corresponding CAS-PDFT results. The molecules are 1,2bibenzyl (i.e., 1,2-diphenylethane), stilbene (i.e., 1,2-diphenylethene), 1,2-bis(diazenyl)ethene, and the bimetallic complex $\left[\mathrm{Mn}\left(\mathrm{NH}_{3}\right)_{4}\right]$ oxamide $\left[\mathrm{Cu}(\mathrm{NH} 3)_{2}\right]^{2+}$, and they are shown in Fig. 1. 


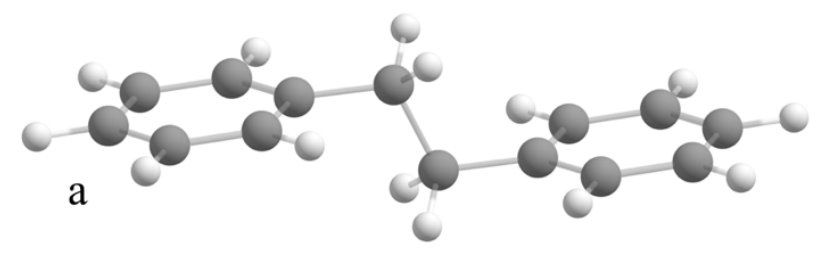

世 (1) O C C $\mathrm{Cu}$

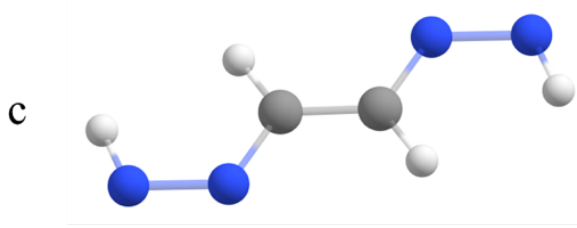

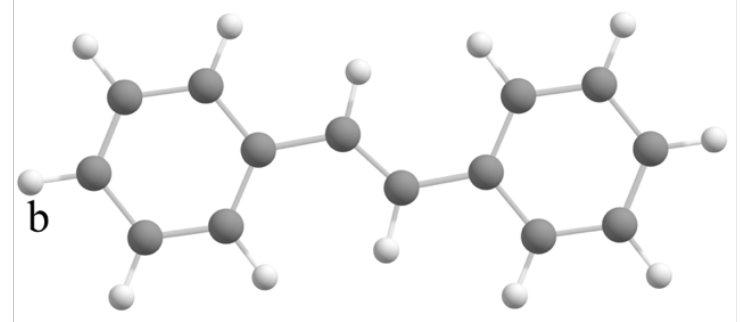

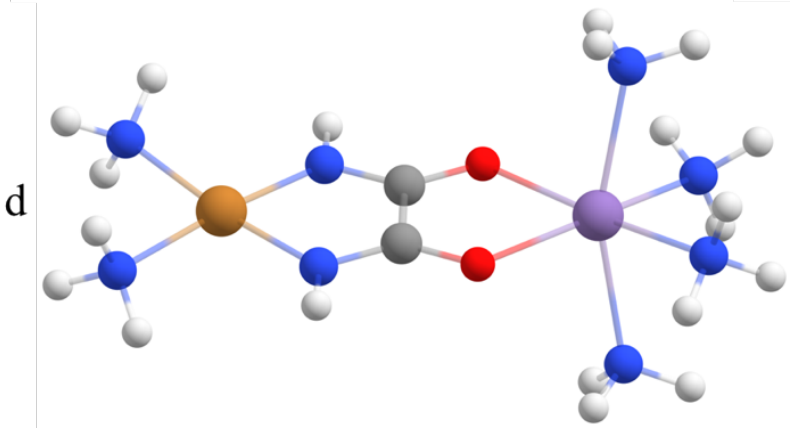

Figure 1: The systems studied in this work: (a) bibenzyl, (b) stilbene, (c) 1,2-bis(diazenyl)ethene, (d) $\left(\left[\mathrm{Mn}\left(\mathrm{NH}_{3}\right)_{4}\right]\right.$ oxamide $\left.\left[\mathrm{Cu}\left(\mathrm{NH}_{3}\right)_{2}\right]^{2+}\right)$.

\section{bibenzyl}

The active space considered for the CASSCF calculation of bibenzyl has 12 active electrons in the $12 \pi$ and $\pi^{*}$ orbitals $(12,12)$. The two phenyl rings in this molecule are connected by a $\mathrm{C}-\mathrm{C}$ single bond. An intuitive LASSCF scheme to decompose this active space is to localize an active subspace of 6 electrons in 6 orbitals on each phenyl ring $(6,6)$. Thus there are two active subspaces and one inactive subspace; note that, as usual in LASSCF calculations, the inactive subspace is delocalized over the whole system. With this scheme, we calculated the lowest singlet and triplet energies as a function of the internal rotation of the central C-C bond by changing the dihedral angle from $55^{\circ}$ to $180^{\circ}$ ). The triplet configuration in the LASSCF wave function was obtained by imposing a triplet spin in one of the $(6,6)$ subspaces while the other was kept as a singlet. 


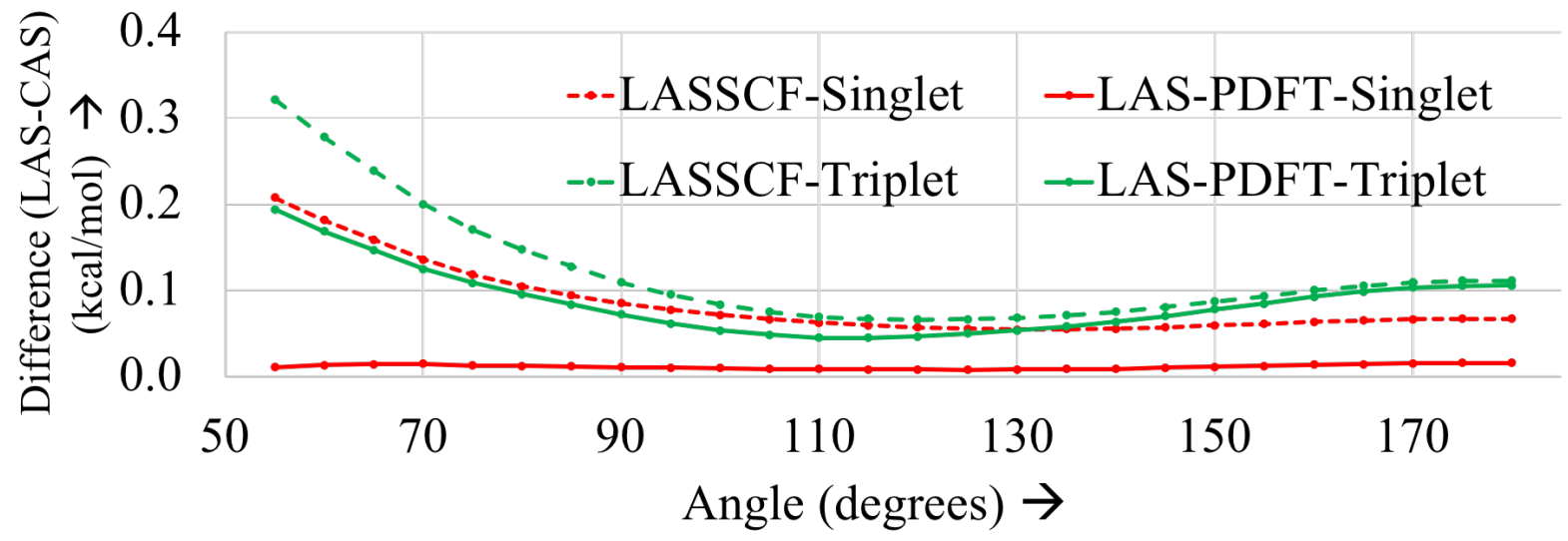

Figure 2: Difference in singlet (in red) and triplet (in green) LASSCF (dotted lines) and LAS-PDFT (solid lines) absolute energies for bibenzyl, computed as the difference with the corresponding CASSCF and CAS-PDFT energies, respectively.

Fig. 2 shows that the difference in LASSCF and LAS-PDFT energies with respect to their CAS equivalents is less than $0.5 \mathrm{kcal} / \mathrm{mol}$ for both the singlet and the triplet states along the entire curve. This confirms that the scheme of unentangling the active space into two subspaces is a reasonable approximation in this case. It is also to be noted that the difference for LAS-PDFT is always smaller than for LASSCF. The strong agreement in the triplet energies in spite of the fragmentation is due to the fact that even in the CASSCF wave function, the two unpaired electrons are localized on one of the phenyl rings itself as seen in the Table S2. Thus, the unentanglement of the subspaces in LASSCF does not cause a heavy energy penalty as it would if the unpaired electrons were delocalized.

This result is particularly appealing because of the significant reduction in the cost of the computation in LASSCF. The number of configuration state functions (CSFs) is 226512 for the singlet and 382239 for the triplet CASSCF wave function, whereas it is 350 and 364 for the singlet and the triplet LASSCF wave functions, respectively. 
We note that bibenzyl is a case where one probably would have anticipated that separating the wholemolecule active space into subspaces would not be harmful and that, although the difference reduces with PDFT, the agreement between CAS-PDFT and LAS-PDFT is largely due to the agreement between the starting wave functions.

\section{Stilbene}

A more difficult test for the theory is a case where unentangling of the active subspaces imposes a high variational penalty on the energies calculated - for example when active orbitals are highly delocalized. An example of such a case is stilbene.

In stilbene the two phenyl rings are connected through a $\mathrm{C}-\mathrm{C}$ double bond and thus the $\pi$ and $\pi^{*}$ orbitals in this molecule are not as localized as in bibenzyl. We use an active space of 10 electrons in 10 orbitals (see Fig. 3) because this has been shown to be a useful active space for this molecule. ${ }^{33}$ Selected calculations using the active space containing all $\pi$ and $\pi^{*}$ orbitals (of 14 electrons in 14 orbitals) are reported in the SI. 

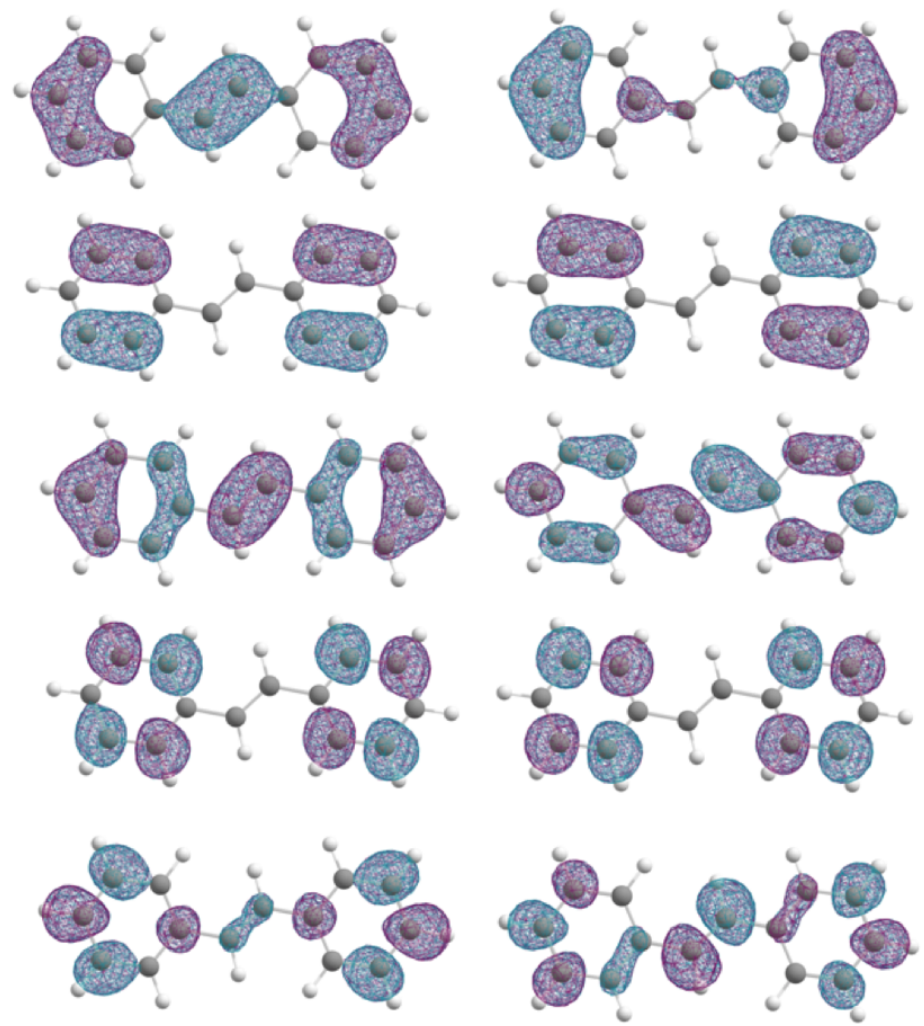

Figure 3: The $(10,10)$ active space for stilbene; shown here are the natural orbitals for a singlet CASSCF calculation at the trans geometry.

Since the molecule is conjugated, the scheme to decompose the active space into subspaces is not as obvious as for bibenzyl. We decomposed it into three active subspaces where a $(4,4)$ subspace is localized on each phenyl ring and a $(2,2)$ subspace on the central $\mathrm{CH}-\mathrm{CH}$ unit. We compare the singlet and triplet energies as the dihedral angle changes from $0^{\circ}$ to $180^{\circ}$, going from cis to trans stilbene. The LASSCF triplet is obtained by imposing a triplet spin on $(2,2)$ subspace. The other possible configuration obtained by imposing a triplet on one of the $(4,4)$ subspaces was found to be higher in energy. 

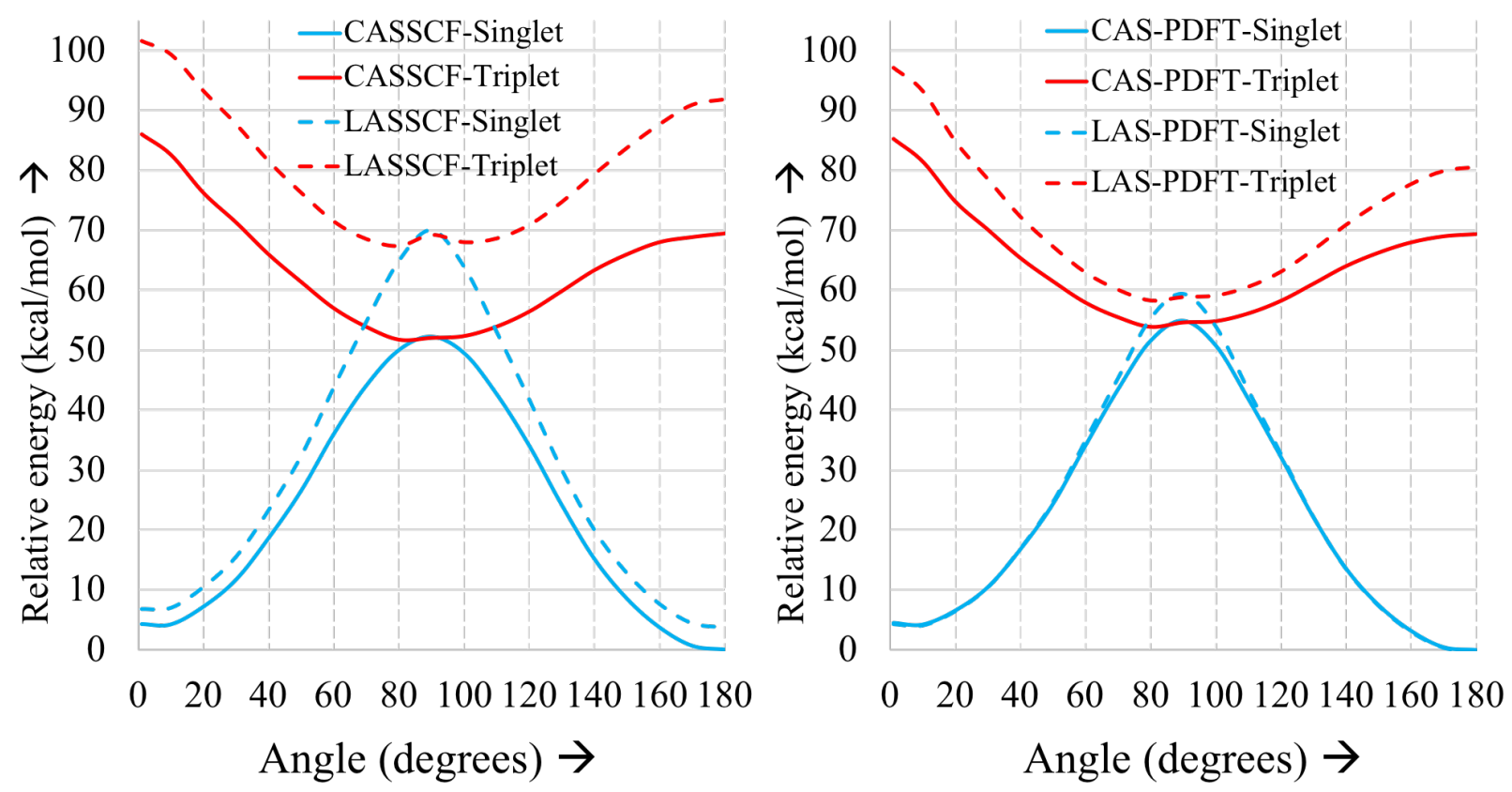

Figure 4: The singlet (blue) and triplet (red) energies (kcal/mol) relative to the CASSCF (left) and CASPDFT (right) singlet for global minimum trans-stilbene calculated using LAS (dashed lines) and CAS (solid lines) wave functions.

Figure 4 shows the CAS and LAS energies along the dihedral scan relative to the singlet CAS energy at the global minimum trans geometry. The triplet CAS energy is also reported relative to the same reference. This shows that LASSCF, in spite of the drastic unentanglement of subspaces, gives a qualitatively accurate description for both spin states. The CASSCF vs. LASSCF energy difference for the triplet is higher than that for the singlet for most geometries. This is can be attributed to the energy penalty imposed by the unentanglement of the active unpaired electrons that would otherwise be more delocalized. The two spin states are nearly degenerate at a dihedral angle of $90^{\circ}$.

The difference in the LASSCF singlet peaks at around $90^{\circ}$ because the wave function becomes more multireference near that geometry as compared to the cis or trans geometries. This is evident from the weight of the dominant (closed-shell singlet) configuration in the CASSCF wave function. It is nearly $91 \%$ at cis and trans geometries, while only $66 \%$ at $90^{\circ}$, where the wave function is more poorly described as a 
product of active subspaces. This is reflected in a higher variational penalty on the LASSCF energy at the $90^{\circ}$ geometry compared to the cis or trans geometries.

The agreement between the LAS-PDFT and CAS-PDFT energies is better than the one between the LASSCF and CASSCF energies for both spin states. Similarly, a faster convergence - with the size of the active space and bond dimension - was detected in previous work for the DMRG-PDFT energy and the CAS-PDFT energy as compared to the DMRG energy and the CASSCF energy. ${ }^{21}$

While the difference between LAS-PDFT and CAS-PDFT is nearly zero for the singlet state at most geometries, some difference still persists at around $90^{\circ}$. To further investigate this difference (and the parent wave functions), we inspected the natural orbitals and their occupancies. At the $90^{\circ}$ geometry there is a near degeneracy in the $\pi$ and $\pi^{*}$ orbitals of the central C-C bond, and the singlet wave function has an openshell character. This can be seen in fig. 5 where the occupancy of both these natural orbitals is close to 1. The LASSCF wave function, which includes the singlet $(2,2)$ localized subspace, shows an open-shell character as well. The natural occupation numbers of these orbitals are reasonably close to those in the CASSCF wave function. The orbitals, however, are significantly more localized.
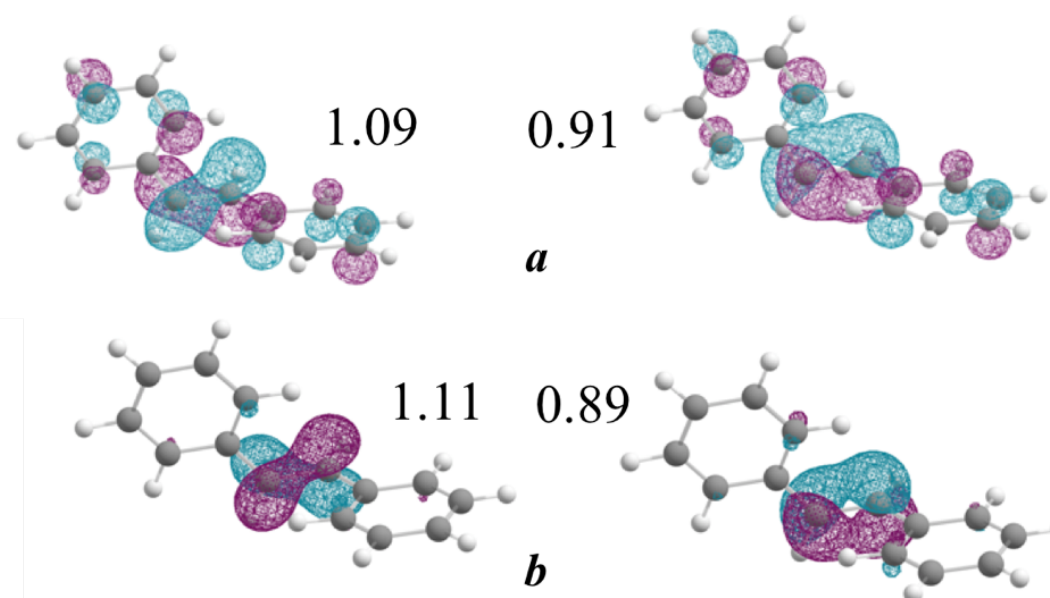

$\boldsymbol{b}$

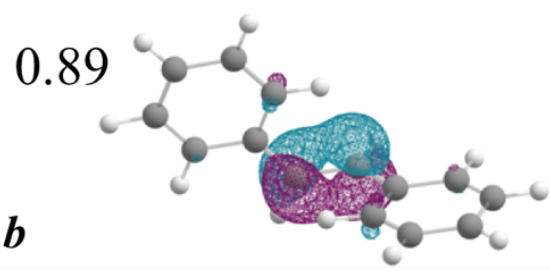

Figure 5: The two natural orbitals from the CASSCF calculation at the $90^{\circ}$ dihedral angle that are mostly singly occupied (top panel $\boldsymbol{a}$ ); the natural orbitals from the $(2,2)$ subspace in the singlet LASSCF calculation (bottom panel $\boldsymbol{b}$ ). 
We also observe that the difference between LAS-PDFT and CAS-PDFT energies for the triplet is higher than that for the singlet. This trend occurs also in the LASSCF versus CASSCF energies. More interesting, however, is the observation that the difference in the triplet curve of LAS-PDFT is lowest at around $90^{\circ}$. To further investigate this, we explored the unpaired density (for the singlet) and the spin density (for the triplet) localized over the three units (two phenyl rings and the $\mathrm{C}_{2} \mathrm{H}_{2}$ group) as seen in fig. 6 . The unpaired density for the triplet (Figure S2) shows a similar behavior to its spin density.
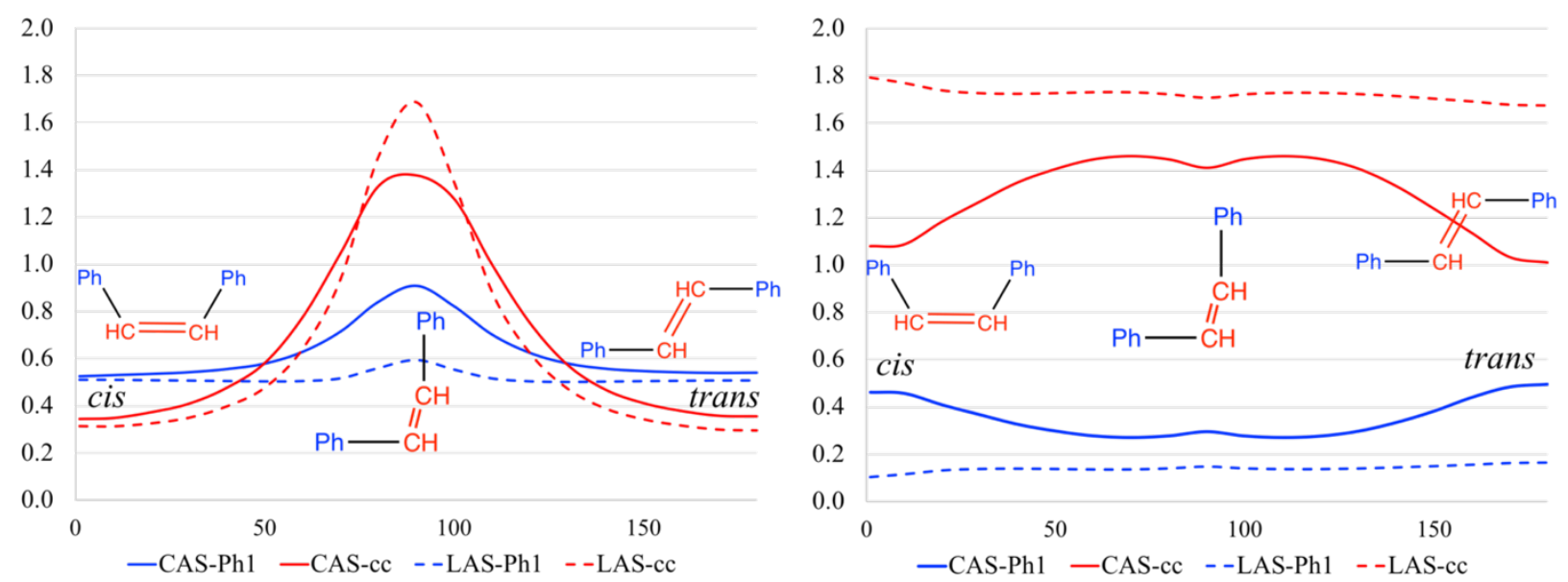

Figure 6: Unpaired density of the singlet (left) and the spin density of the triplet (right) wave functions localized on the phenyl (red) and CH-CH (blue) units in CASSCF (solid lines) and LASSCF (dotted lines) calculations

We notice that the LAS unpaired density for the singlet is quantitatively similar to the CAS unpaired density at the equilibrium geometries, and the difference is greatest around the $90^{\circ}$ angle. This also demonstrates that the LAS wave function does capture the open-shell character at the $90^{\circ}$ angle, but it has the unpaired electrons localized on the $\mathrm{C}-\mathrm{C}$ bond. In the triplet case, however, since we impose a triplet on $(2,2)$ subspace that is localized on the $\mathrm{CH}-\mathrm{CH}$ unit for all the geometries, nearly the entire spin density of 1.8 electrons is localized in the $\mathrm{CH}-\mathrm{CH}$ unit at all the angles in LASSCF. The "correct" CASSCF densities over each unit 
are not the same for all the angles. The cis and trans geometries have only about 1 electron worth of spin localized over the $\mathrm{CH}-\mathrm{CH}$ unit while the other two phenyl rings have about 0.5 electrons each.

We can correlate the disagreement between the LASSCF and CASSCF energies at specific geometries for both spin states to the disagreement in the corresponding spin (for triplet) and unpaired (for singlet) densities that are delocalized in the entire molecule in CASSCF, while are restricted on the central $\mathrm{CH}-\mathrm{CH}$ unit in LASSCF. When the LASSCF spin/unpaired densities are closer to the CASSCF ones, LAS-PDFT agrees with CAS-PDFT even more strikingly than LASSCF with CASSCF, perhaps indicating that LAS-PDFT energies are more immune to the changes in density as compared to the LASSCF energies. An analysis of the breakdown of the components of the PDFT energies (SI Section S1) shows that the absolute difference $\left(\mathrm{E}_{\mathrm{ot}, \mathrm{LAS}}-\mathrm{E}_{\mathrm{ot}, \mathrm{CAS}}\right)$ in the on-top energy is similar, $\sim 5 \mathrm{kcal} / \mathrm{mol}$, for both spins, while the energy terms from the reference MCSCF wave function cause the higher difference in the triplet.

We also tested the effect of changing the basis sets $(6-31 G(d)$ and $6-31+G(d))$ and the size of the active space $(\mathrm{a}(14,14)$ active space) for the dihedral scan of stilbene (SI section S2). The $(14,14)$ active space, which includes all the $\pi$ and $\pi^{*}$ orbitals of stilbene, is decomposed in three active subspaces with a $(6,6)$ subspace on each phenyl ring and a $(2,2)$ subspace on the $\mathrm{CH}-\mathrm{CH}$ unit. This leads to an even more drastic reduction in the cost. While the $(14,14)$ CASSCF singlet and triplet have 2760615 and 5010005 CSFs respectively, the corresponding LASSCF wavefunctions have only 353 and $351 \mathrm{CSF}$. While the difference in LASSCF and LAS-PDFT are independent of the basis set size, the disagreement between LAS and CAS energies increases for a larger $(14,14)$ active space, as expected based on the greater reduction in the CSFs. Even in this case the LAS-PDFT difference did not increase as much as the LASSCF. 


\section{Concerted dissociation of the N-N bonds in 1,2-bis(diazenyl)ethene}

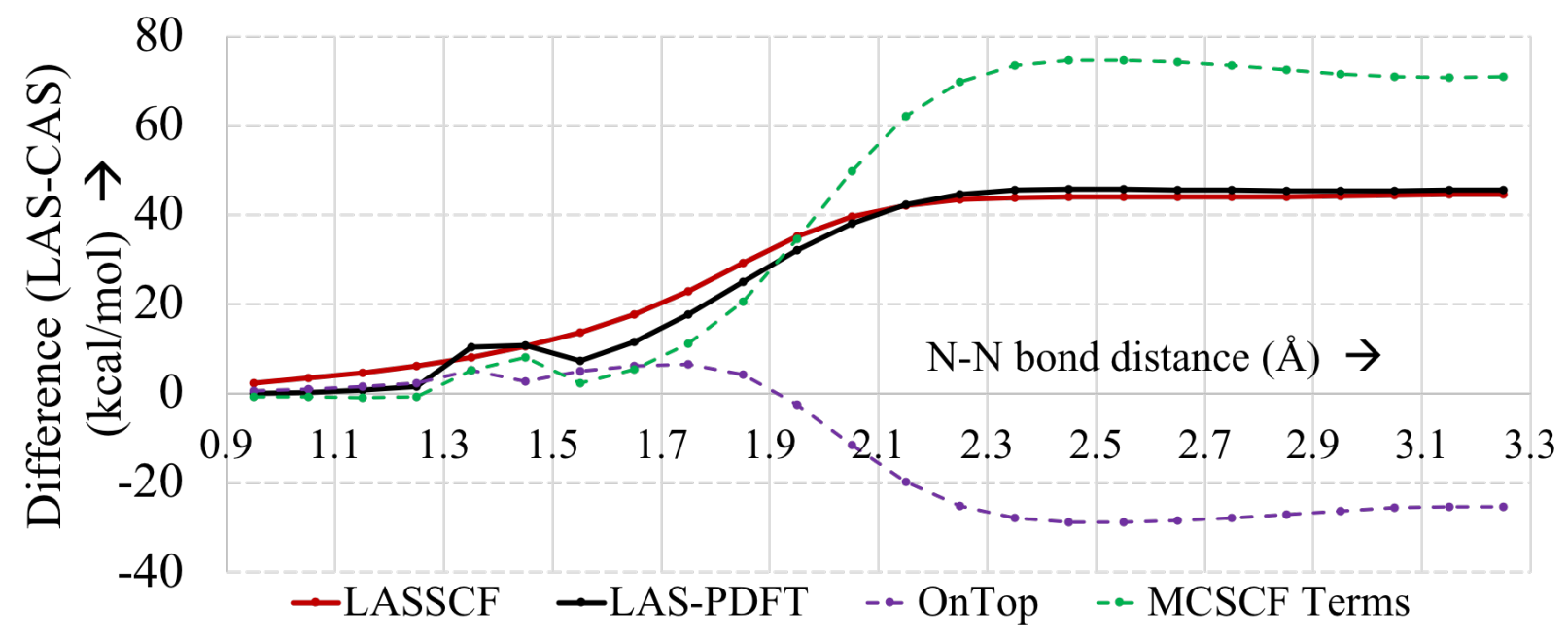

Figure 7: Difference (kcal/mol) between the LASSCF and CASSCF absolute energies (red) and LAS-PDFT and CAS-PDFT absolute energies (black) along the dissociation curves and break down of the LAS-PDFT difference in its components.

We explored the performance of LAS-PDFT for the concerted dissociation of 1,2-bis(diazenyl)ethene, previously studied using LASSCF. ${ }^{24}$ This is an example where LASSCF fails to quantitatively predict the dissociation energy because there are two $\mathrm{N}=\mathrm{N}$ double bonds connected by a central $\mathrm{C}=\mathrm{C}$ double bond, so overall the $\pi$ system is highly delocalized. Following our previous work, the $(10,10)$ active space of this molecule is decomposed in three localized active subspaces with a $(4,4)$ subspace on each $\mathrm{N}_{2} \mathrm{H}$ unit and a $(2,2)$ subspace on the $\mathrm{C}_{2} \mathrm{H}_{2}$ unit. Figure 7 shows the energy difference along the dissociation reaction coordinate between LASSCF and CASSCF ( $\left.\mathrm{E}_{\mathrm{LASSCF}}-\mathrm{E}_{\mathrm{CASSCF}}\right)$ and between LAS-PDFT and CAS-PDFT $\left(\mathrm{E}_{\mathrm{LAS}-\mathrm{PDFT}}-\mathrm{E}_{\mathrm{CAS}-\mathrm{PDFT}}\right)$. As the multireference character of the wave function increases with the N-N bond distance, $\left(\mathrm{E}_{\mathrm{LASSCF}}-\mathrm{E}_{\mathrm{CASSCF}}\right)$ and $\left(\mathrm{E}_{\mathrm{LAS}-\mathrm{PDFT}}-\mathrm{E}_{\mathrm{CAS}-\mathrm{PDFT}}\right)$ are about $45 \mathrm{kcal} / \mathrm{mol}$. This was further investigated by plotting the differences in the components of the PDFT energies - namely the on-top energy and the MCSCF terms (sum of kinetic energy, nuclear electron attraction, and classical Coulomb repulsion). The $45 \mathrm{kcal} / \mathrm{mol}$ difference at dissociation can be decomposed into the difference in the on-top energy (-25 
$\mathrm{kcal} / \mathrm{mol})$ and in the MCSCF wave function part $(70 \mathrm{kcal} / \mathrm{mol})$. Further decomposition of these differences is provided in section S4. This shows that both components of energy have a significant difference. The LAS wave function itself is highly approximate at the dissociation limit, and thus it does not serve as a good reference for PDFT.

\section{Spin state ordering in bimetallic compounds}

Predicting the spin-state energy gaps of metal complexes with several metal centers is a challenge for electronic structure theories. In many complexes the metal centers have a high degree of intraatomic electron correlation due to the low-lying virtual d-orbitals being localized around them, but the correlation between two metal centers is not always high. In such cases partitioning the active space results in a substantial reduction of the computational cost without affecting the quality of the results, and, when several metals are present, only partitioning methods may be affordable. We studied the spin state ordering of a bimetallic complex containing a $\mathrm{Cu}$ and $\mathrm{Mn}$ atom, and we showed that LASSCF is able to qualitatively predict the spin ladder in agreement with CASSCF. ${ }^{24}$
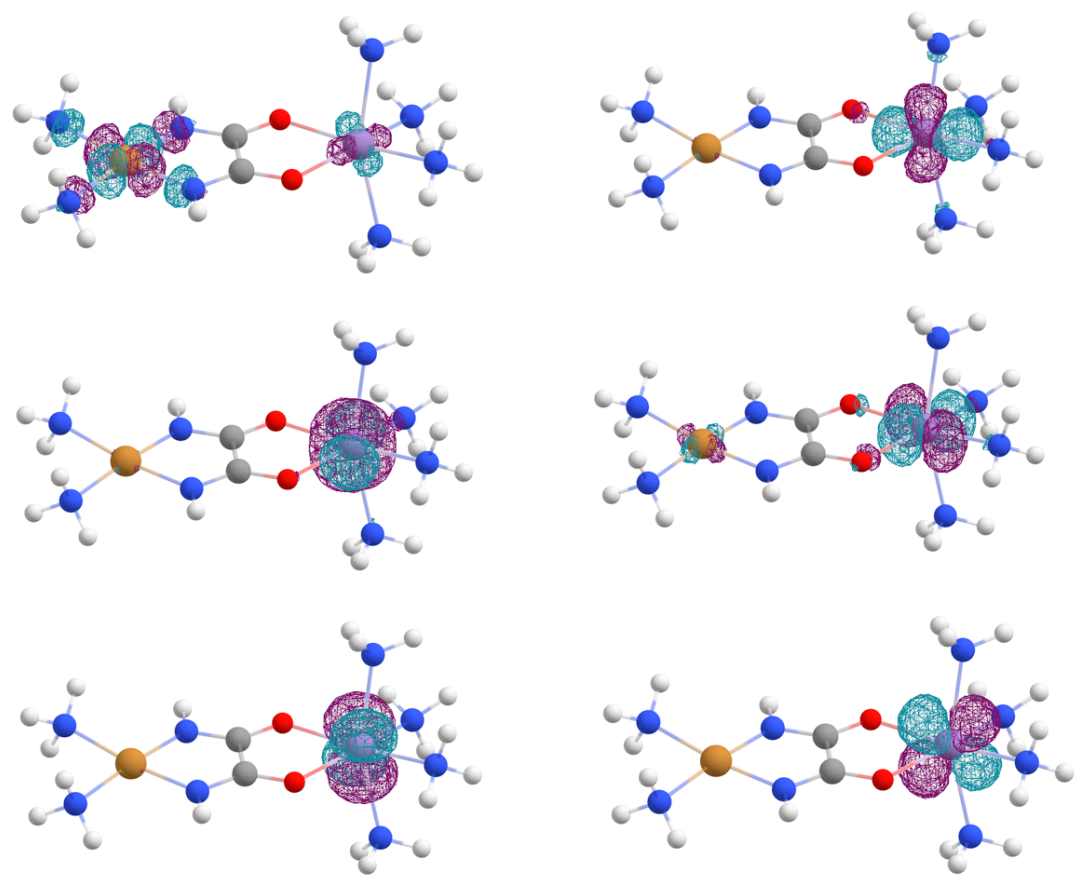

Figure 8: The active orbitals in the minimal active space of the bimetallic complex 
The minimal active space (see Fig. 8) of the six electrons in the five 3d Mn orbitals and one electron in the $\mathrm{Cu} 3 \mathrm{~d}$ orbitals is considered. The LAS wave function has two active subspaces - one with 5 electrons in 5 orbitals localized on the $\mathrm{Mn}$ and the other with 1 electron in 1 orbital localized on the $\mathrm{Cu}$. (This is not intended to be an ideal active space for capturing the majority of electron correlation). The energies for all possible spin states are reported relative to the CASSCF septet state. Since the LAS wave function is not necessarily a spin eigenfunction in these cases, we classify the wave functions based on the expectation value of the spin projection operator, because $M_{S}$ (defined above) is still a good quantum number while $S$ is no longer a good quantum number.

Table 1: Energy differences $(\mathrm{kcal} / \mathrm{mol})$ for different spin states and local spin orientations relative to the respective septet CASSCF or CASPT2 energies, calculated using different methods.

\begin{tabular}{|c|c|c|c|c|c|}
\hline $\begin{array}{l}\text { Spin } \\
\left(\mathrm{m}_{\mathrm{s}}\right)\end{array}$ & CASSCF & CAS-PDFT & $\begin{array}{l}\text { Local spin } \\
\text { orientation }\end{array}$ & LASSCF & LAS-PDFT \\
\hline Septet & 0.0 (ref) & 0.0 (ref) & ferromagnetic & 0.0 & 0.0 \\
\hline \multirow{2}{*}{ Quintet } & \multirow{2}{*}{0.0} & \multirow{2}{*}{-0.1} & anti-ferromagnetic & 0.0 & 0.0 \\
\hline & & & ferromagnetic $^{a}$ & 71.5 & 43.1 \\
\hline \multirow{2}{*}{ Triplet } & \multirow{2}{*}{70.6} & \multirow{2}{*}{42.3} & anti-ferromagnetic & 71.5 & 43.1 \\
\hline & & & ferromagnetic $^{a}$ & 96.6 & 55.8 \\
\hline Singlet & 96.6 & 55.8 & anti-ferromagnetic & 96.6 & 55.8 \\
\hline
\end{tabular}

${ }^{a}$ Ferromagnetic coupling for a quintet and triplet spin involves quartet and a doublet respectively imposed on the $(5,5)$ subspace and a doublet with the same direction of spin polarization on the $(1,1)$ subspace.

LASSCF captures the near degeneracy of the septet and the quintet seen at the CASSCF level and also reproduces the relative energies of the higher-energy spin states, namely the triplet and the singlet. Consequently, LAS-PDFT reproduces the CAS-PDFT gaps with a difference much lower than one $\mathrm{kcal} / \mathrm{mol}$.

A key advantage of the LAS-PDFT method is that it allows us to compute electron correlation beyond the LASSCF needed for reliable energies of the spin states and also the relative energies of spin couplings that are not accessible (or separable) using CASSCF wave functions. The relative energetics of the ferro- and 
antiferromagnetic states demonstrate that the true quintet and triplet ground states are almost certainly dominated by antiferromagnetic interactions between the two metal centers. The LAS form of the wave function exposes this information straightforwardly to the user, and the LAS-PDFT extension incorporates the effects of dynamic electron correlation into the analysis.

\section{Concluding Remarks}

The LASSCF algorithm can be used to obtain a good reference wave function for post-MCSCF calculations, like PDFT. Not only does the LAS-PDFT method account for more correlation than the LASSCF itself, but it also reduces the disagreement between the CAS and LAS energies. In other words, MC-PDFT is in most cases more immune to the unentangling of the active space than is MCSCF. This expands the opportunity to use PDFT for systems for which the reference CASSCF calculation is not affordable. In addition to the favorable scaling, LASSCF also provides us with greater control on the local spin in different subspaces. While the current implementation does not guarantee that any arbitrary spin coupling will result in an overall spin eigenfunction, the different LAS wave functions provide a possible starting point for spinadapting the wave function. Analysis of the spin density and the unpaired density in the various subspaces elucidates the effects of unentangling the active subspaces on the wave functions.

Supporting Information

See supplementary material for further discussion referred in the manuscript as well as absolute energies, coordinates and sample input files.

Notes

The authors declare no competing financial interest.

ACKNOWLEDGMENTS 
This work was supported by the U.S. Department of Energy, Office of Basic Energy Sciences, Division of Chemical Sciences, Geosciences, and Biosciences under Award DE-FG02-17ER16362.

\section{REFERENCES:}

1. Löwdin, P.-O., Correlation Problem in Many-Electron Quantum Mechanics I. Review of Different Approaches and Discussion of Some Current Ideas. Adv. Chem. Phys 1958, 207-322.

2. Chakraborty, I.; Bodurtha, K. J.; Heeder, N. J.; Godfrin, M. P.; Tripathi, A.; Hurt, R. H.; Shukla, A.; Bose, A., Massive electrical conductivity enhancement of multilayer graphene/polystyrene composites using a nonconductive filler. ACS Appl. Mater. Interfaces 2014, 6 (19), 16472-16475.

3. Evangelista, F. A., Perspective: Multireference coupled cluster theories of dynamical electron correlation. J. Chem. Phys. 2018, 149 (3), 030901.

4. Szalay, P. G.; Muller, T.; Gidofalvi, G.; Lischka, H.; Shepard, R., Multiconfiguration self-consistent field and multireference configuration interaction methods and applications. Chem. Rev. 2012, 112 (1), 108-181.

5. Park, J. W.; Al-Saadon, R.; MacLeod, M. K.; Shiozaki, T.; Vlaisavljevich, B., Multireference electron correlation methods: Journeys along potential energy surfaces. Chem. Rev. 2020.

6. Handy, N. C.; Cohen, A. J., Left-right correlation energy. Mol. Phys. 2001, 99 (5), 403412.

7. Boguslawski, K.; Tecmer, P.; Legeza, O.; Reiher, M., Entanglement measures for singleand multireference correlation effects. J. Phys. Chem. Lett. 2012, 3 (21), 3129-3135.

8. Roos, B. O.; Taylor, P. R.; Si, P. E. M.; others, A complete active space SCF method (CASSCF) using a density matrix formulated super-CI approach. Chem. Phys. 1980, 48 (2), 157173.

9. Andersson, K.; Malmqvist, P.-Å.; Roos, B. O., Second-order perturbation theory with a complete active space self-consistent field reference function. J. Chem. Phys. 1992, 96 (2), 12181226.

10. Vancoillie, S.; Zhao, H.; Tran, V. T.; Hendrickx, M. F. A.; Pierloot, K., Multiconfigurational Second-Order Perturbation Theory Restricted Active Space (RASPT2) Studies on Mononuclear First-Row Transition-Metal Systems. J. Chem. Theory Comput 2011, 7 (12), 3961-3977.

11. Loos, P.-F.; Scemama, A.; Jacquemin, D., The Quest for Highly Accurate Excitation Energies: A Computational Perspective. J. Phys. Chem. Lett. 2020, 11 (6), 2374-2383.

12. Li Manni, G.; Carlson, R. K.; Luo, S.; Ma, D.; Olsen, J.; Truhlar, D. G.; Gagliardi, L., Multiconfiguration pair-density functional theory. J. Chem. Theory Comput 2014, 10 (9), 36693680 .

13. Wilbraham, L.; Verma, P.; Truhlar, D. G.; Gagliardi, L.; Ciofini, I., Multiconfiguration pair-density functional theory predicts spin-state ordering in iron complexes with the same accuracy as complete active space second-order perturbation theory at a significantly reduced computational cost. J. Phys. Chem. Lett. 2017, 8 (9), 2026-2030.

14. Sand, A. M.; Truhlar, D. G.; Gagliardi, L., Efficient algorithm for multiconfiguration pairdensity functional theory with application to the heterolytic dissociation energy of ferrocene. $J$. Chem. Phys. 2017, 146 (3), 034101. 
15. Dong, S. S.; Gagliardi, L.; Truhlar, D. G., Excitation spectra of retinal by multiconfiguration pair-density functional theory. Phys. Chem. Chem. Phys. 2018, 20 (10), 72657276.

16. Gagliardi, L.; Truhlar, D. G.; Li Manni, G.; Carlson, R. K.; Hoyer, C. E.; Bao, J. L., Multiconfiguration pair-density functional theory: A new way to treat strongly correlated systems. Acc. Chem. Res. 2016, 50 (1), 66-73.

17. Presti, D.; Stoneburner, S. J.; Truhlar, D. G.; Gagliardi, L., Full Correlation in a Multiconfigurational Study of Bimetallic Clusters: Restricted Active Space Pair-Density Functional Theory Study of [2Fe-2S] Systems. J. Phys. Chem. C 2019, 123 (18), 11899-11907.

18. Ghosh, S.; Cramer, C. J.; Truhlar, D. G.; Gagliardi, L., Generalized-active-space pairdensity functional theory: an efficient method to study large, strongly correlated, conjugated systems. Chem. Sci. 2017, 8 (4), 2741-2750.

19. Bao, J. L.; Odoh, S. O.; Gagliardi, L.; Truhlar, D. G., Predicting bond dissociation energies of transition-metal compounds by multiconfiguration pair-density functional theory and secondorder perturbation theory based on correlated participating orbitals and separated pairs. J. Chem. Theory Comput 2017, 13 (2), 616-626.

20. Li, S. J.; Gagliardi, L.; Truhlar, D. G., Extended separated-pair approximation for transition metal potential energy curves. J. Chem. Phys. 2020, 152 (12), 124118.

21. Sharma, P.; Bernales, V.; Knecht, S.; Truhlar, D. G.; Gagliardi, L., Density matrix renormalization group pair-density functional theory (DMRG-PDFT): singlet-triplet gaps in polyacenes and polyacetylenes. Chem. Sci. 2019, 10 (6), 1716-1723.

22. Zhou, C.; Gagliardi, L.; Truhlar, D. G., Multiconfiguration Pair-Density Functional Theory for Iron Porphyrin with CAS, RAS, and DMRG Active Spaces. J. Phys. Chem. A 2019, 123 (15), 3389-3394.

23. Presti, D.; Truhlar, D. G.; Gagliardi, L., Intramolecular Charge Transfer and Local Excitation in Organic Fluorescent Photoredox Catalysts Explained by RASCI-PDFT. J. Phys. Chem. C 2018, 122 (22), 12061-12070.

24. Hermes, M. R.; Pandharkar, R.; Gagliardi, L., Variational Localized Active Space SelfConsistent Field Method. J. Chem. Theory Comput 2020, 16 (8), 4923-4937.

25. Hermes, M. R.; Gagliardi, L., Multiconfigurational Self-Consistent Field Theory with Density Matrix Embedding: The Localized Active Space Self-Consistent Field Method. J. Chem. Theory Comput 2019, 15 (2), 972-986.

26. Mazziotti, D. A., Contracted Schrödinger equation: Determining quantum energies and two-particle density matrices without wave functions. Phys. Rev. A 1998, 57 (6), 4219.

27. Sun, Q.; Chan, G. K.-L., Exact and Optimal Quantum Mechanics/Molecular Mechanics Boundaries. J. Chem. Theory Comput 2014, 10 (9), 3784-3790.

28. Sun, Q.; Berkelbach, T. C.; Blunt, N. S.; Booth, G. H.; Guo, S.; Li, Z.; Liu, J.; McClain, J. D.; Sayfutyarova, E. R.; Sharma, S.; Wouters, S.; Chan, G. K. L., PySCF: the Python-based simulations of chemistry framework. 2017; Vol. 8, pp e1340-e1340.

29. Chang, S.; Davidson, E.; Vincow, G., Theory of the Hyperfine Splittings of Pi-Electron Free Radicals. III. Methyl Radical in a Pyramidal Configuration: Temperature Dependence of the Hyperfine Splittings. J. Chem. Phys. 1970, 52 (11), 5596-5606.

30. Staroverov, V. N.; Davidson, E. R., Distribution of effectively unpaired electrons. Chemical Physics Letters 2000, 330 (1-2), 161-168.

31. Staroverov, V. N.; Davidson, E. R., Transition regions in the Cope rearrangement of 1,5hexadiene and its cyano derivatives. J. Am. Chem. Soc. 2000, 122 (30), 7377-7385. 
32. Takatsuka, K.; Fueno, T.; Yamaguchi, K., Distribution of odd electrons in ground-state molecules. Theor. Chim. Acta 1978, 48 (3), 175-183.

33. Gagliardi, L.; Orlandi, G.; Molina, V.; Malmqvist, P.-Å.; Roos, B., Theoretical study of the lowest 1BU states of trans-stilbene. J. Phys. Chem. A 2002, 106 (32), 7355-7361. 